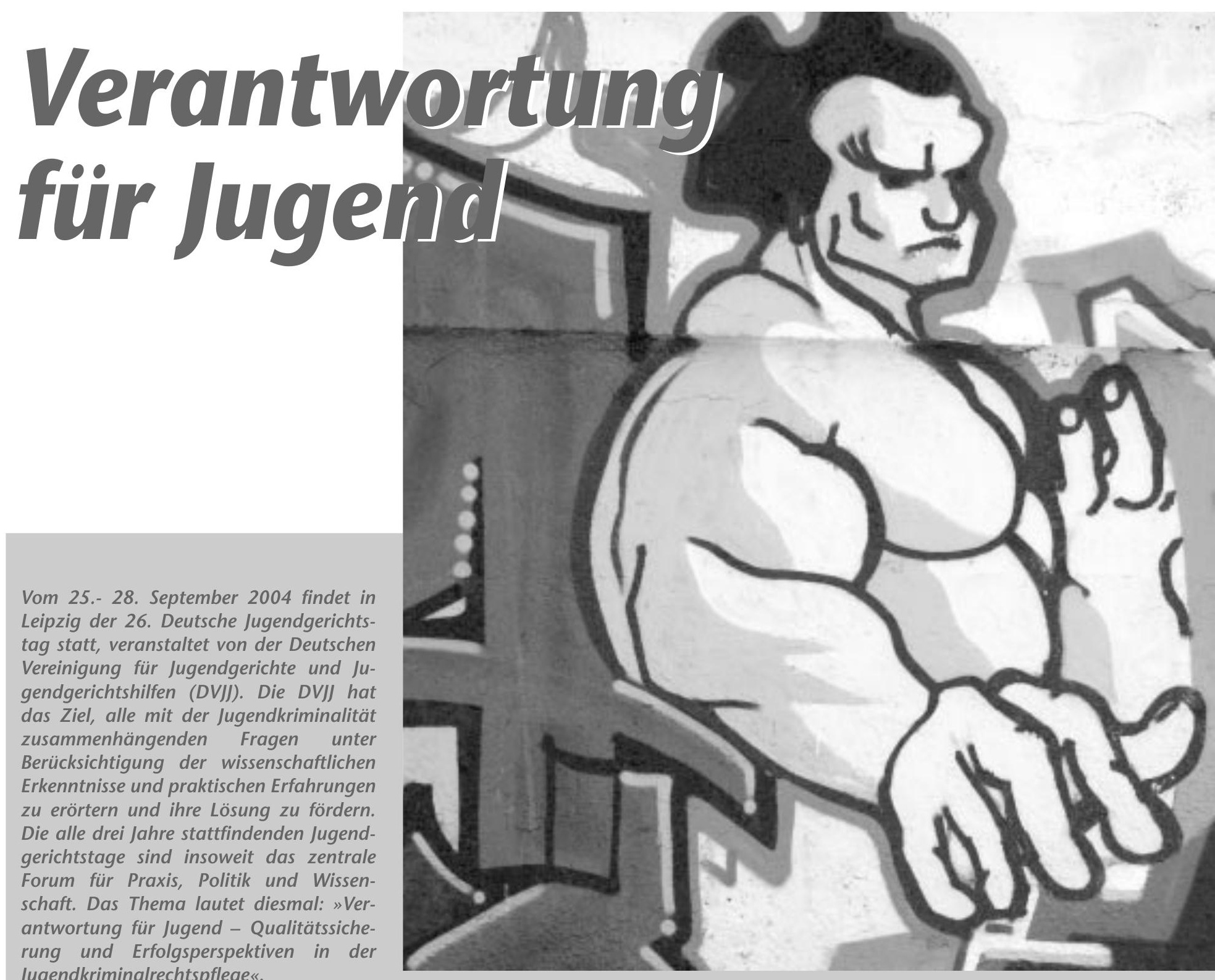

Jugendkriminalrechtspflege".

Sowohl im Eröffnungsreferat von Winfried Hassemer als auch im Schlussreferat von Leoluca Orlando (ehemaliger Bürgermeister von Palermo) wird deutlich werden, dass "Verantwortung für Jugend " praktisch der kleinste gemeinsame Nenner aller in der Jugendkriminalrechtspflege Tätigen ist. Wolfgang Heinz wird die neuesten wissenschaftlichen Untersuchungen zur Qualitätssicherung und den Erfolgsperspektiven vorstellen. "Was können wir? « und "Was wissen wir? « - um diese Fragestellungen der beiden Foren gruppieren sich jeweils acht Arbeitskreise. Eine Podiumsdiskussion mit internationalen Gästen sowie der Markt der Möglichkeiten runden den inhaltlichen Teil des Jugendgerichtstages $a b$.

Die praxisorientierten wissenschaftlichen Themen können kaum aktueller und vielfältiger sein, geht es doch beispielsweise um die Frage der Grenzen der Erziehung speziell auch bei sogenannten Intensivtätern. Aus Kostengründen sind zahlreiche Angebote der Jugendhilfe gefährdet, so dass Jugendrichterinnen und -richter mitunter auf die traditionellen stationären Sanktionen des Jugendstrafrechts beschränkt sind. Das grundlegende Problem einer verbesserten Kommunikation und Kooperation der am Jugendstrafverfahren beteiligten Berufsgruppen mit der Forderung, unterschiedliche Perspektiven und Kompetenzen verstehen und anerkennen zu können, wird ebenso behandelt wie die Frage nach Alternativen zur Justiz und Alternativen in der Justiz. Dabei geht es um Konfliktbewältigung durch Mediation und Täter-OpferAusgleich. Die neuen ambulanten Maßnahmen müssen sich die Fragestellung gefallen lassen, ob es sich um Qualität oder Wildwuchs handelt. Im Bereich des Jugendstrafvollzuges geht es dann u.a. darum, ob das zu erwartende Jugendstrafvollzugsgesetz chancenreiche Rahmenbedingungen schaffen wird. Lebenswelt- und Sozialraumorientierung führen zu einer Überprüfung von Zuständigkeiten in der sozialen Arbeit, und zwar auch im Verhältnis von Spezialistentum und Allround-Zuständigkeit. Auf 
Grund der Erkenntnisse zu Zusammenhängen zwischen Schulschwänzen und Jugendkriminalität ergeben sich neue Kooperationen zwischen Schule und Jugendhilfe. Der entsprechende Arbeitskreis heißt: "Konfliktfeld Schule: Zwischen Bildung, Ausgrenzung und Kontrolle».

Die Frage "Was wissen wir « dient nicht nur dazu zu klären, wo Wissenslücken durch empirische Untersuchungen zu schließen sind, sondern provoziert auch die selbstkritische Einschätzung, was wir wissen könnten und sollten. Ergebnisse der Wirkungsforschung beispielsweise des Sherman-Reports und des Düsseldorfer Gutachtens sind auf ihre praktische Bedeutung für Jugendhilfe und Justiz zu überprüfen. Der sich abzeichnende Trend zu mehr Härte ("Neue Rigidität«) ließe sich mit Hil- fe der aktuellen Ergebnisse empirischer Sanktionsforschung vielleicht sogar stoppen. Zumindest würden die Vorschläge der 2. Jugendstrafrechtsreform-Kommission und des 64. Deutschen Juristentages in Berlin 2002 wieder in das kriminalpolitische Blickfeld kommen. Eine Evaluation von Praxisprojekten (»Ohne Evaluation ist die Praxis blind «) könnte die Qualität der Arbeit im Schnittstellenbereich von Jugendhilfe und Justiz verbessern. Dabei geht es dann auch um die Diagnostik in der Sozialen Arbeit. Angesprochen sind die Methode der sozialpädagogischen Diagnosen und die psychosoziale Diagnostik. Die methodischen Ansätze, aber vor allem auch die rechtsstaatlichen Grenzen der Kriminalprävention sind vorzustellen und zu diskutieren.
Die EU-Osterweiterung wird Chancen für grenzüberschreitende Modellprojekte der Jugendarbeit eröffnen. Hier stellen sich aber auch neue Herausforderungen für Jugendhilfe und Justiz. In einem ganz anderen Bereich gibt es neue Erkenntnisse, die erstmals auf dem Jugendgerichtstag präsentiert werden und zwar über Jugendliche zwischen Medienkompetenz und medialer Verwahrlosung.

Nicht nur wegen der aktuellen Themen, zu erwartender neuer Erkenntnisse und interessanter Diskussionen sollte eine Teilnahme am Jugendgerichtstag in Leipzig erwogen werden, sondern vor allem unter dem Aspekt VERANTWORTUNG FÜR JUGEND.

Bernd-Rüdeger Sonnen

\section{Rückzug der Jugendhilfe aus dem Jugendstrafverfahren?}

\section{Kooperation versus Rollenrückzug und Rollenverwischung}

Prof. Dr. Heribert Ostendorf

\section{Sozialpädagogische Sanktionen Mangelware}

\begin{abstract}
m Jahre 1994 wurde unter der Leitung von Frieder Dünkel eine bundesweite Umfrage zum Angebot ambulanter Maßnahmen nach dem JGG durch die JGH durchgeführt. Damals sagten $26 \%$ der Jugendrichter, es fehle an einem Angebot sozialer Trainingskurse. 11\% sagten, das Angebot sei nicht ausreichend (Dünkel/Geng/ Kirstein, Soziale Trainingskurse, S. 217). Fast $20 \%(19,9)$ gaben an, dass kein Angebot für den TOA bestehe, $15,4 \%$ hielten das Angebot für nicht ausreichend. Besser wurde das Angebot für Betreuungsweisungen eingeschätzt. Obwohl Schleswig-Holstein damals besser als der Bundesdurchschnitt abschnitt, hat eine neue Untersuchung aus meiner Forschungsstelle für das Jahr 2003 folgendes Ergebnis gebracht:
\end{abstract}

$45 \%$ der Jugendrichter in Schleswig-Holstein halten das Angebot des sozialen Trainingskurses, $18 \%$ das Angebot der Betreuungsweisung und
64\% das Angebot für eine Unterbringung in einer Erziehungseinrichtung für nicht ausreichend. Als Hauptgrund für das fehlende Angebot wird die fehlende bzw. unklare Kostenträgerschaft genannt (s. demnächst Körner, Kostentragungspflicht im Jugendstrafverfahren). Die Situation hat sich offensichtlich in den letzten Jahren verschlechtert, nicht nur in Schleswig-Holstein.

Im Rahmen der ambulanten Sanktionen werden im Jugendstrafrecht ganz überwiegend Arbeitsmaßnahmen und Geldbußen angeordnet. Deren erzieherischer Wert hält sich bekanntlich in Grenzen.

\section{Kooperationsanspruch und Kooperationswirklichkeit}

Nicht nur die Umsetzung jugendgerichtlicher Sanktionen, wie sie der Gesetzgeber im JGG den Jugendgerichten angeboten hat, findet nur z. T. statt, auch die Kooperation und Kommunikation vorher, d. h. im Ermittlungsverfahren sowie in der Hauptverhandlung leiden Not.

Bezeichnend für die Situation vor Ort ist die Zeitungsmeldung (siehe Seite 113):

Dass eine Zusammenarbeit der am Jugendstrafverfahren Beteiligten sachlich für einen vernünftigen Umgang mit jungen Straftätern geboten ist, darüber besteht Einigkeit, auch zur Vermeidung von überflüssiger Arbeit. Über die Verpflichtungen im Einzelnen gibt es Streit, z. T. auch blanke Unkenntnis. So wird die vom Gesetzgeber vorgesehene Verpflichtung für die Jugendgerichtshilfe, auch während des Strafvollzugs tätig zu bleiben, d. h. mit dem verurteilten Jugendlichen Verbindung zu halten und an der Wiedereingliederung mitzuwirken ( $\$ 38$ Abs. 2 S. 8 JGG), in der Praxis weitgehend nicht befolgt: »Während des Vollzuges bleiben sie mit dem Jugendlichen in Verbindung und nehmen sich seiner Wiedereingliederung in die Gemeinschaft an.« Für die Zeit der U-Haft hat die JGH sogar un- 